\title{
EULER-LAGRANGE EQUATIONS WITH 3-DIMENSIONAL REAL NUMBER SPACE ON AN ALMOST PARACONTACT MANIFOLD
}

\section{ZEKI KASAP}

Pamukkale University

Faculty of Education

Kinikli Campus

Denizli

Turkey

e-mail: zkasap@pau.edu.tr

\begin{abstract}
We consider Euler-Lagrange equations on almost paracontact manifolds. It is well known that the geometry of almost contact manifolds is a natural extension in the odd dimensional case of almost Hermitian geometry. A Hermitian manifold is a complex manifold with a Hermitian metric on its holomorphic tangent space. Also, the contact geometry as symplectic geometry has large and comprehensive applications in physics, geometrical optics, classical mechanics, thermodynamics, geometric quantization, and applied mathematics. On the other hand, one way of solving problems in classical and analytical mechanics is through use of the Euler-Lagrange equations. The purpose of the present paper is to solve the problems of classical mechanics with 3-dimensional real number space on an almost paracontact manifold by using Euler-Lagrange equations.
\end{abstract}

2010 Mathematics Subject Classification: 34N05, 53D05, 53D10, 70H03.

Keywords and phrases: symplectic manifold, contact manifold, mechanical system, dynamic equation, Lagrangian formalism.

Received December 26, 2015; Revised February 17, 2016

(C) 2016 Scientific Advances Publishers 


\section{Introduction}

Symplectic and contact geometry are such theories that naturally emerged from the mathematical description of classical physics. They were revolutionized in the early 1980s with the discovery of new rigidity phenomena and properties satisfied by these geometric structures. Ever since, they have been very useful in the development of many areas of mathematics and modern mathematical physics. Contact manifolds are the natural framework for geometric optics. Contact structures arise naturally on energy levels of autonomous Hamiltonian systems. Many basic concepts, such as symplectic reduction, Hamilton-Jacobi equations, Legendrian and Lagrangian submanifolds were developed in this context and continue to play an important role to date.

Modern science has found a lot of applications in differential geometry and mathematical physics. One of the most important applications for differential geometry is on geodesics. A geodesic is the shortest route between defined two points. The Lagrangian formulation is an important tramplen from which develops another useful formulation of classical mechanics known as the Hamiltonian formulation develops. One way of finding geodesics is the Euler-Lagrange equations. It is shown that Lagrangian mechanics is a very important tool for differential geometry and analytical mechanics. They have a simple method to describe the model for mechanical systems. There are studies about contact manifold and mechanical systems. A summary of these studies is shown below:

Tripathi et al. [1] introduced the concept of $\varepsilon$-almost paracontact manifolds, and in particular, of $\varepsilon$-para-Sasakian manifolds. Srivastava et al. [2] submitted the concept of ( $(\varepsilon)$-almost paracontact manifolds. They showed that some typical identities for curvature tensor and Ricci tensor of ( $\varepsilon$ )-para Sasakian manifolds are obtained and studied the properties of $\varepsilon-S$ paracontact metric manifold. Girtu [3] showed that $K$ induces an almost 2-paracontact Riemannian structure on $T_{0}^{*} M$, whose restriction to the guratrix bundle $K=\{(x, p) \mid K(x, p)=1\}$ is an almost paracontact 
structure. Ahmad and Jun [4] defined a semi-symmetric non-metric connection in an almost $r$-paracontact Riemannian manifold and they considered submanifolds of an almost $r$-paracontact Riemannian manifold endowed with a semi-symmetric non-metric connection. Also, in [5], [6], Kupeli Erken studied 3-dimensional normal almost paracontact metric manifolds. Cappelletti Montano et al. [7] studied paracontact metric manifolds for which the Reeb vector field of the underlying contact structure satisfies a nullity condition. Kupeli Erken and Murathan [9] completed a study of three-dimensional paracontact metric $(\widetilde{\kappa}, \widetilde{\mu}, \widetilde{v})$-manifolds. They focus on some curvature properties by considering the class of paracontact metric $(\kappa, \mu, v)$-manifolds under a condition. Welyczko [8] showed that the curvature and torsion of slant Frenet curves in 3-dimensional normal almost paracontact metric manifolds. Manev and Staikova [10] gave a classification with eleven basic classes of almost paracontact Riemannian manifolds of type $(n, n)$ with respect to the covariant derivative of the $(1,1)$-tensor of the almost paracontact structure. Pandey and Tiwari [11] showed that a recurrent special $(\in)$-para Sasakian manifold with $\eta$ as 1 -form of recurrence is Einstein manifold. Shukla and Verma [12] introduced the notion of paracomplex paracontact pseudo-Riemannian submersions from almost para-Hermitian manifolds onto almost paracontact metric manifolds. Gunduzalp and Sahin [13] first defined the concept of paracontact semiRiemannian submersions between almost paracontact metric manifolds, then they provided an example and showed that the vertical and horizontal distributions of such submersions are invariant with respect to the almost paracontact structure of the total manifold. Bucki [14] showed that for an almost $r$-paracontact manifold of $P$-Sasakian type there exists a product submanifold. Acet et al. [15] studied canonical paracontact connection on a para-Sasakian manifold. Ahmad et al. [16] defined a quarter symmetric semi-metric connection in an almost $r$-paracontact Riemannian manifold and considered invariant, non-invariant, and antiinvariant hypersurfaces of an almost $r$-paracontact Riemannian manifold with that connection. Nakova and Zamkovoy [17] considered almost paracontact pseudo-Riemannian manifolds with indefinite metric $g$, 
which is it compatible with almost paracontact structure and it satisfies the condition. Kasap and Tekkoyun [18] obtained Lagrangian and Hamiltonian formalism for mechanical systems using para/pseudoKähler manifolds, representing an interesting multidisciplinary field of research. Kasap [19] introduced that the Weyl-Euler-Lagrange and Weyl-Hamilton equations on $\mathbb{R}_{n}^{2 n}$, which is a model of tangent manifolds of constant W-sectional curvature. Tekkoyun [20] found paracomplex analogue of Euler-Lagrange and Hamiltonian equations. Also, he gave the geometric results on the related mechanic systems.

\section{Preliminaries}

In this study, $M$ is a manifold and $T M$ is a tangent bundle. $F(T M)$, $\chi(T M)$, and $\Lambda^{1}(T M)$ denote the set of complex functions on $T M$, the set of complex vector fields on $T M$, and the set of complex 1-forms on $T M$, respectively. Also, $X$ and $Y$ are any vector fields on $M$.

Definition 1. Let $M$ be a differentiable manifold of dimension $(2 n+1)$, and suppose $J$ is a differentiable vector bundle isomorphism $J: T M \rightarrow T M$ such that $J_{x}: T_{x} M \rightarrow T_{x} M$ is a (almost) complex structure for $T_{x} M$, i.e., $J^{2}=I$, where $I$ is the identity (unit) operator on $V$. Then $J$ is called an almost paracomplex structure for the differentiable manifold $M$. A manifold with a fixed almost paracomplex structure is called an (almost) paracomplex manifold. Where $J^{2}=J \circ J$, and $I$ is the identity operator on $T M$.

Definition 2. A Riemannian metric $g$ is compatible metric with these structure tensors if

$$
g(\varphi X, \varphi Y)=-g(X, Y)+\eta(X) \eta(Y),
$$

and $(\varphi, \xi, \eta, g)$ is called an almost paracontact metric structure. Note also that $g(X, \xi)=\eta(X)$. If it satisfies $d \eta(X, Y)=g(\varphi X, Y)$ for any vector fields $X, Y$ on $M$, then $(M, \varphi, \xi, \eta, g)$ is called a paracontact Riemannian manifold. 
Let $M$ be a $(2 n+1)$ dimensional smooth manifold. Let $\varphi$ be a $(1,1)$-type tensor field, $\xi$ is a vector field, and $\eta$ is a 1 -form on $M$. Then the $(\varphi, \xi, \eta)$ is called an almost paracontact structure on $M$, if the following conditions are satisfied:

$$
\left.\begin{array}{ll}
\text { (1) } & \varphi(\xi)=0, \\
\text { (2) } & \eta \circ \varphi(X)=0, \\
\text { (3) } \eta(\xi)=1, \\
\text { (4) } \varphi^{2} X=X-\eta(X) \xi .
\end{array}\right\}
$$

If we donote by $\{\eta\}^{\perp}$ the subbundle of the tangent bundle $T(M)$ of $M$ consisting of all vectors annihilated by $\eta$, then the tensor field $\varphi$ induces an almost paracomplex structure on each fibre of $\{\eta\}^{\perp}$. The pair $(M, \varphi, \xi, \eta)$ is called an almost paracontact manifold.

Definition 3. Let $M$ be an almost contact manifold and define an almost complex structure $J$ on $M \times \mathbb{R}$ by

$$
J\left(X+f \frac{d}{d t}\right)=\varphi X-f \xi(X)+\eta(X) \frac{d}{d t},
$$

for any vector field $X$ on $M$, where $f$ is a $\mathbb{C}^{\infty}$ function on $M \times \mathbb{R}$. An almost contact structure is said to be normal if $J$ is integrable. An almost contact metric structure $(\varphi, \xi, \eta, g)$ is called Sasakian if furthermore

$$
\left(\nabla_{X} \varphi\right)(Y)=\eta(Y) X-g(X, Y) \xi
$$

where $\nabla$ is the Levi-Civita connection of $g$.

Definition 4. An immediate consequence of the definition of the almost paracontact structure is that the endomorphism $\varphi$ has rank $2 n$, $\varphi \xi=0$ and $\eta \circ \varphi=0$. If a manifold $M^{(2 n+1)}$ with $(\varphi, \xi$, $\eta)$-structure admits a pseudo-Riemannian metric $g$ such that (1), then we say that 
$M^{(2 n+1)}$ has an almost paracontact metric structure and $g$ is called compatible metric. Any compatible metric $g$ with a given almost paracontact structure is necessarily of signature $(n+1, n)$. Setting $Y=\xi$, we have $\eta(X)=g(X, \xi)([21],[22])$.

\section{Nijenhuis Tensor}

A celebrated theorem of Newlander and Nirenberg [23] says that an almost (para) complex structure is a (para) complex structure if and only if its Nijenhuis tensor or torsion vanishes.

Theorem 1. An almost paracontact structure $(\varphi, \xi, \eta)$ is said to be normal, if the Nijenhuis tensor $N_{J}$ of almost paracontact structure $J$ is defined as

$$
N_{J}(X, Y)=[J, J](X, Y)=[J X, J Y]+J^{2}[X, Y]-J[J X, Y]-J[X, J Y] .
$$

The almost paracontact structure $J$ on $M$ is integrable if and only if the tensor $N_{J}$ vanishes identically, where $N_{J}$ is defined on two vector fields $X$ and $Y$. The tensor $(2,1)$ is called the Nijenhuis tensor (5). If $N_{J}=0$, then the almost paracontact structure is called paracontact or integrable.

\section{Paracontact Manifold}

Let $M$ be a differential manifold, $T M$ its tangent bundle, and $\xi \subset T M$ a field of hyperplanes on $M$ a smooth sub-bundle of codimension 1. Here the terms differential and smooth are used synonymously with $\mathbb{C}^{\infty}$. Locally, $\xi$ can be written as the kernel of a non-vanishing differential 1-form $\alpha$. A 1 -form $\alpha$ defined globally on $M$ with $\xi=\operatorname{ker} \alpha$ can be found if and only if $\xi$ is coorientable, which is equivalent to 
saying that the quotient line bundle $T M / \xi$ is trivial. The 1 -form $\alpha$ is determined by $\xi$ up to multiplication by a smooth function $f: M \rightarrow \mathbb{R}^{*}$ or, if the coorientation of $\xi$ has been fixed, by a function taking positive real values only.

Definition 5. Let $M$ be a smooth manifold of dimension $(2 n+1)$. A contact structure on $M$ is a hyperplane field $\xi \subset T M$, whose (locally) defining 1 -form $\alpha$ has the property that the $(2 n+1)$-form $\alpha \wedge(d \alpha)^{n}$ is non zero, i.e., a volume form, on its domain of definition.

Observe that the condition $\alpha \wedge(d \alpha)^{n} \neq 0$ is indeed a property of $\xi$ and independent of the choice of defining 1 -form $\alpha$, since $(f \alpha) \wedge d(f \alpha)^{n}$ $=f^{n+1} \alpha \wedge(d \alpha)^{n}$.

Definition 6. A pair $(M, \xi)$ consisting of an odd-dimensional manifold $M$ and a contact structure $\xi$ on $M$ is called a contact manifold.

Definition 7. A 1 -form as in Definition 5 defined globally on $M$, is called a contact form on $M$.

Definition 8. If $g(X, \varphi Y)=d \eta(X, Y)$, where $d \eta(X, Y)=\frac{1}{2}(X \eta(Y)$ $-Y \eta(X)-\eta([X, Y]))$, then $\eta$ is a paracontact form and the almost paracontact metric manifold $(M, \varphi, \eta, g)$ is said to be paracontact metric manifold [21].

Example 1. Let $\mathbb{R}^{3}$ be the 3-dimensional real number space with a coordinate system $(x, y, z)$. We define $\eta=d z-y d x, \xi=\frac{\partial}{\partial z}$

$$
\begin{aligned}
& \text { (1) } \varphi\left(\frac{\partial}{\partial x}\right)=-\frac{\partial}{\partial x}-y \frac{\partial}{\partial z} \\
& \text { (2) } \varphi\left(\frac{\partial}{\partial y}\right)=-\frac{\partial}{\partial y} \\
& \text { (3) } \varphi\left(\frac{\partial}{\partial z}\right)=0
\end{aligned}
$$


Then, $(\varphi, \xi, \eta)$ is an almost paracontact structure $[1,2]$ in $\mathbb{R}^{3}$. Here, instead of $J$ conformal structure representing the structure of $\varphi$ will be used. Now, we denote the structure $(\varphi)$ of the holomorphic property:

$$
\begin{aligned}
& \text { (1) } \varphi^{2}\left(\frac{\partial}{\partial x}\right)=\varphi \circ \varphi\left(\frac{\partial}{\partial x}\right)=\varphi\left(-\frac{\partial}{\partial x}-y \frac{\partial}{\partial z}\right) \\
& =-\varphi\left(\frac{\partial}{\partial x}\right)-y \varphi\left(\frac{\partial}{\partial z}\right)=\frac{\partial}{\partial x}+y \frac{\partial}{\partial z}, \\
& \text { that is, } \quad \varphi^{2}\left(\frac{\partial}{\partial x}\right)=I d-\eta \otimes \xi \text {, } \\
& \text { (2) } \varphi^{2}\left(\frac{\partial}{\partial y}\right)=\varphi \circ \varphi\left(\frac{\partial}{\partial y}\right)=\frac{\partial}{\partial y}, \varphi^{2}\left(\frac{\partial}{\partial y}\right)=I d \text {, } \\
& \text { (3) } \varphi^{2}\left(\frac{\partial}{\partial z}\right)=0 \text {. }
\end{aligned}
$$

Above (1) and (2) structures of (7) are paracontact. Also, (3) structure is tangent (exactly).

\section{J-Holomorphic Functions}

Definition 9. Let $U$ be an open set of $\mathbb{C}$, and $f: U \rightarrow \mathbb{C}$ a continuously differentiable mapping. We can write $f=u+i v=u(x, y)$ $+i v(x, y)$, where $x+i y$ and $u+i v$ are complex coordinates on the domain and target, respectively. The function $f$ is said to be holomorphic if the resulting differential $d f=f_{*}: \mathbb{R}^{2} \rightarrow \mathbb{R}^{2}$ is complex linear.

As defined above, this means that the Jacobian matrix of partial derivatives commutes with multiplication by complex $i$, so that

$$
\left(\begin{array}{cc}
u_{x} & u_{y} \\
v_{x} & v_{y}
\end{array}\right)\left(\begin{array}{cc}
0 & -1 \\
1 & 0
\end{array}\right)=\left(\begin{array}{cc}
0 & -1 \\
1 & 0
\end{array}\right)\left(\begin{array}{cc}
u_{x} & u_{y} \\
v_{x} & v_{y}
\end{array}\right),
$$

and one obtains the Cauchy-Riemann equations $u_{x}=v_{y}, v_{x}=-u_{y}$ [24]. 


\section{Almost-Hermitian Manifold}

Definition 10. A Hermitian manifold is the complex analogue of a Riemannian manifold. Specifically, a Hermitian manifold is a complex manifold with a smoothly varying Hermitian inner product on each (holomorphic) tangent space. One can also define a Hermitian manifold as a real manifold with a Riemannian metric that preserves a complex structure.

Definition 11. Let $M$ be an almost-Hermitian manifold, that is a $2 n$-dimensional Riemannian manifold admitting an orthogonal almost complex structure $J$. We shall often indicate the Riemannian metric $g$ by $\langle\cdot, \cdot\rangle$. An associated metric $g$ and $J$ is the fundamental 2 -form $\omega$ defined by $\omega(X, Y)=\langle J X, Y\rangle=-\langle X, J Y\rangle, X, Y \in T_{m} M$, whose skewsymmetry follows from the equation $\langle J X, J Y\rangle=\langle X, Y\rangle$ [25].

\section{Symplectic Manifold}

Definition 12. A symplectic vector space is a vector space $V$ over a field $F$ (for example, the real numbers $\mathbb{R}$ ) equipped with a symplectic bilinear form $\omega: V \times V \rightarrow F$. The bilinear form $\omega$ is said to be symplectic, if it is (a) alternating: $\omega(v, v)=0$ for all $v \in V$, (b) nondegenerate: if $\omega(u, v)=0$ for all $v \in V$, then $u=0$.

Definition 13. A symplectic manifold is a smooth manifold $M$ equipped with a closed nondegenerate differential 2-form $(\omega)$ called the symplectic form. The study of symplectic manifolds is called symplectic geometry or symplectic topology. Symplectic manifolds arise naturally in abstract formulations of classical mechanics and analytical mechanics as the cotangent bundles of manifolds, e.g., in the Hamiltonian and Lagrangian formulation of classical mechanics, which provides one of the major motivations for the field. The set of all possible configurations of a system is modelled as a manifold, and this manifold's cotangent bundle describes the phase space of the system. 
The basic example of an almost complex symplectic manifold is standard Euclidean space $\left(\mathbb{R}^{2 n}, \omega_{0}\right)$ with its standard almost complex structure $J_{0}$ obtained from the usual identification with $\mathbb{C}_{n}$. Thus, one sets $z_{j}=x_{2 j-1}+i x_{2 j}$ for $j=1, \ldots, n$ and defines $J_{0}$ by

$$
J_{0}\left(\partial_{2 j-1}\right)=\partial_{2 j}, \quad J_{0}\left(\partial_{2 j}\right)=-\partial_{2 j-1},
$$

where $\partial_{j}=\partial / \partial x_{j}$ is the standard basis of $T_{x} \mathbb{R}^{2 n}[26]$.

\section{8. (Euler)-Lagrange Dynamics Equations}

A dynamic system with a finite number $n$ degrees of freedom can be described by real functions of time $q^{i}(t)(i=1,2, \ldots, n)$ which, together with the derivatives image $\dot{q}^{i}$, uniquely determine its state at any moment of time $t$. The collection of all values of $q^{i}$ is called the configuration space $M$ of the system. In the simplest case, $M$ is a Euclidean space $\mathbb{R}^{n}$.

Definition 14. Let $M$ be an $n$-dimensional manifold and $T M$ its tangent bundle with canonical projection $\tau_{M}: T M \rightarrow M$. TM is called the phase space of velocities of the base manifold $M$. Let $L: T M \rightarrow \mathbb{R}$ be a differentiable function on $T M$ and is called the Lagrangian function.

Lemma 1. The closed 2-form on a vector field and 1-form reduction function on the phase space defined of a mechanical system is equal to the differential of the energy function 1-form of the Lagrangian mechanical systems $[27,28]$.

Definition 15. We consider closed 2 -form on $T M$ such that $\Phi_{L}=-d\left(\mathbf{d}_{J} L\right)$ and $\mathbf{i}_{\xi}$ is 2 -form reduction function that reduces the 1-form. Consider the equation

$$
\mathbf{i}_{\Omega} \Phi_{L}=d E_{L}
$$


where the semispray $\Omega$ is a vector field. We shall see that for motion in a potential, $E_{L}=V(L)-L$ is an energy function and $V=J(\Omega)$ is a Liouville vector field. Here $d E_{L}$ denotes the differential of $E$. We shall see that Equation (10) under a certain condition on $\Omega$ is the intrinsical expression of the Euler-Lagrange equations of motion. This equation is named as Euler-Lagrange dynamical equation.

Definition 16. The triple $\left(T M, \Phi_{L}, \Omega\right)$ is known as Euler-Lagrangian system on the tangent bundle $T M$.

Definition 17. Consider the following combination of the kinetic $(T)$ and potential energies $(V), L=T-V$. This is called the Lagrangian. There is a minus sign in the definition (a plus sign would simply give the total energy). In the problem of a mass on the end of a spring $T=\frac{1}{2} m \dot{x}^{2}$, $V=\frac{1}{2} k x^{2}, L=\frac{1}{2} m \dot{x}^{2}-\frac{1}{2} k x^{2}$. Now write

$$
\frac{d}{d t}\left(\frac{\partial L}{\partial \dot{x}}\right)-\frac{\partial L}{\partial x}=0, \dot{x}=\frac{d x}{d t}
$$

This equation (11) is called the Euler-Lagrange (E-L) equation [25-27].

\section{Euler-Lagrangian Equations}

Using Lemma 1, we can be obtained Euler-Lagrange equations for classical and analytical mechanics on 3-dimensional real number space and it is shown by $(T M, g, \varphi)$. Let $\Omega$ be the vector field determined (to be similar [20]) on $(T M, g, \varphi)$ for the calculation of the equation (10) and $(x, y, z)$ be coordinate functions,

$$
\Omega=X \frac{\partial}{\partial x}+Y \frac{\partial}{\partial y}+Z \frac{\partial}{\partial z}, \quad X=\dot{x}=y, \quad Y=\dot{y}=z, \quad Z=\dot{z} .
$$

Then the vector field defined by

$$
\varphi(\Omega)=\varphi\left(X \frac{\partial}{\partial x}+Y \frac{\partial}{\partial y}+Z \frac{\partial}{\partial z}\right)=X\left(-\frac{\partial}{\partial x}-y \frac{\partial}{\partial z}\right)+Y\left(-\frac{\partial}{\partial y}\right)
$$


is thought to be Liouville vector field on almost paracontact manifold $(T M, g, \nabla, \varphi) . \Phi_{L}=-d\left(\mathbf{d}_{\varphi} L\right)$ is the closed 2 -form given by (10) such that

$$
\mathbf{d}=\frac{\partial}{\partial x} d x+\frac{\partial}{\partial y} d y+\frac{\partial}{\partial z} d z, \mathbf{d}_{\varphi}: F(M) \rightarrow \wedge^{1} M
$$

and

$$
\begin{aligned}
& \mathbf{d}_{\varphi}=i_{\varphi} \mathbf{d}-\mathbf{d} i_{\varphi}, \\
& \mathbf{d}_{\varphi}=\varphi(\mathbf{d})=\varphi\left(\frac{\partial}{\partial x}\right) d x+\varphi\left(\frac{\partial}{\partial y}\right) d y+\varphi\left(\frac{\partial}{\partial z}\right) d z .
\end{aligned}
$$

Also, the vertical differentiation $\mathbf{d}_{\varphi}$ is given by $d$ is the usual exterior derivation. Here, we can account Euler-Lagrange equations for classical and quantum mechanics on 3-dimensional real number space $(T M, g, \varphi)$. We get the equations given by

$$
\mathbf{d}_{\varphi}=\left(-\frac{\partial}{\partial x}-y \frac{\partial}{\partial z}\right) d x+\left(-\frac{\partial}{\partial y}\right) d y
$$

Let account $\Phi_{L}$,

$$
\begin{aligned}
\Phi_{L}= & -d\left(\mathbf{d}_{\varphi} L\right) \\
= & \left(-\frac{\partial^{2} L}{\partial x \partial x}-y \frac{\partial^{2} L}{\partial x \partial z}\right) d x \wedge d x-\frac{\partial^{2} L}{\partial x \partial y} d y \wedge d x \\
& +\left(-\frac{\partial^{2} L}{\partial y \partial x}-y \frac{\partial^{2} L}{\partial y \partial z}\right) d x \wedge d y-\frac{\partial^{2} L}{\partial y \partial y} d y \wedge d y \\
& +\left(-\frac{\partial^{2} L}{\partial z \partial x}-y \frac{\partial^{2} L}{\partial z \partial z}\right) d x \wedge d z-\frac{\partial^{2} L}{\partial z \partial y} d y \wedge d z
\end{aligned}
$$


and then we find using

$$
\begin{gathered}
(f \wedge g)(v)=f(v) g-g(v) f \\
\left(d x_{i} \wedge d x_{j}\right)\left(\frac{\partial}{\partial x_{k}}\right) \\
=d x_{i} \frac{\partial}{\partial x_{k}} d x_{j}-d x_{j} \frac{\partial}{\partial x_{k}} d x_{i} \\
=\frac{\partial x_{i}}{\partial x_{k}} d x_{j}-\frac{\partial x_{j}}{\partial x_{k}} d x_{i} \\
\frac{\partial x_{i}}{\partial x_{k}}=0, \quad \frac{\partial x_{i}}{\partial x_{i}}=1
\end{gathered}
$$

and

$$
\begin{aligned}
i_{\Omega} \Phi_{L} & =\Phi_{L}(\Omega) \\
& =X\left(-\frac{\partial^{2} L}{\partial x \partial x}-y \frac{\partial^{2} L}{\partial x \partial z}\right) d x-X\left(-\frac{\partial^{2} L}{\partial x \partial x}-y \frac{\partial^{2} L}{\partial x \partial z}\right) d x+X\left(\frac{\partial^{2} L}{\partial x \partial y}\right) d y \\
& +X\left(-\frac{\partial^{2} L}{\partial y \partial x}-y \frac{\partial^{2} L}{\partial y \partial z}\right) d y+X\left(-\frac{\partial^{2} L}{\partial z \partial x}-y \frac{\partial^{2} L}{\partial z \partial z}\right) d z-Y\left(\frac{\partial^{2} L}{\partial x \partial y}\right) d x \\
& -Y\left(-\frac{\partial^{2} L}{\partial y \partial x}-y \frac{\partial^{2} L}{\partial y \partial z}\right) d x-Y\left(\frac{\partial^{2} L}{\partial y \partial y}\right) d y+Y\left(\frac{\partial^{2} L}{\partial y \partial y}\right) d y-Y\left(\frac{\partial^{2} L}{\partial z \partial y}\right) d z \\
& -Z\left(-\frac{\partial^{2} L}{\partial z \partial x}-y \frac{\partial^{2} L}{\partial z \partial z}\right) d x+Z\left(\frac{\partial^{2} L}{\partial z \partial y}\right) d y
\end{aligned}
$$

Also, the Lagrangian energy function of system is

$$
V=\varphi(\Omega)=\varphi\left(X \frac{\partial}{\partial x}+Y \frac{\partial}{\partial y}+Z \frac{\partial}{\partial z}\right)=X\left(-\frac{\partial}{\partial x}-y \frac{\partial}{\partial z}\right)+Y\left(-\frac{\partial}{\partial y}\right)+0
$$

Moreover,

$$
E_{L}=\varphi(\Omega) L-L=X\left(-\frac{\partial L}{\partial x}-y \frac{\partial L}{\partial z}\right)+Y\left(-\frac{\partial L}{\partial y}\right)-L
$$


and the differential of $E_{L}$ is

$$
\begin{aligned}
d E_{L}= & X\left(-\frac{\partial^{2} L}{\partial x \partial x}-y \frac{\partial^{2} L}{\partial x \partial z}\right) d x-Y\left(\frac{\partial^{2} L}{\partial x \partial y}\right) d x \\
& -\frac{\partial L}{\partial x} d x+X\left(-\frac{\partial^{2} L}{\partial y \partial x}-y \frac{\partial^{2} L}{\partial y \partial z}\right) d y-Y\left(\frac{\partial^{2} L}{\partial y \partial y}\right) d y-\frac{\partial L}{\partial y} d y \\
& +X\left(-\frac{\partial^{2} L}{\partial z \partial x}-y \frac{\partial^{2} L}{\partial z \partial z}\right) d z-Y\left(\frac{\partial^{2} L}{\partial z \partial y}\right) d z-\frac{\partial L}{\partial z} d z
\end{aligned}
$$

Using (10), we get equations as follows:

$$
\begin{aligned}
& -X\left(-\frac{\partial^{2} L}{\partial x \partial x}-y \frac{\partial^{2} L}{\partial x \partial z}\right) d x-Y\left(-\frac{\partial^{2} L}{\partial y \partial x}-y \frac{\partial^{2} L}{\partial y \partial z}\right) d x \\
& -Z\left(-\frac{\partial^{2} L}{\partial z \partial x}-y \frac{\partial^{2} L}{\partial z \partial z}\right) d x+X\left(\frac{\partial^{2} L}{\partial x \partial y}\right) d y+Y\left(\frac{\partial^{2} L}{\partial y \partial y}\right) d y \\
= & -\frac{\partial L}{\partial x} d x-\frac{\partial L}{\partial y} d y .
\end{aligned}
$$

We find the following equations:

$$
\begin{aligned}
& \text { dif1. }-X\left(-\frac{\partial^{2} L}{\partial x \partial x}-y \frac{\partial^{2} L}{\partial x \partial z}\right)-Y\left(-\frac{\partial^{2} L}{\partial y \partial x}-y \frac{\partial^{2} L}{\partial y \partial z}\right) \\
& -Z\left(-\frac{\partial^{2} L}{\partial z \partial x}-y \frac{\partial^{2} L}{\partial z \partial z}\right)+\frac{\partial L}{\partial x}=0, \\
& {\left[X \frac{\partial}{\partial x}+Y \frac{\partial}{\partial y}+Z \frac{\partial}{\partial z}\right]\left(\frac{\partial L}{\partial x}\right)} \\
& +\left[X \frac{\partial}{\partial x}+Y \frac{\partial}{\partial y}+Z \frac{\partial}{\partial z}\right]\left(y \frac{\partial L}{\partial z}\right)+\frac{\partial L}{\partial x}=0, \\
& \Omega\left(\frac{\partial L}{\partial x}\right)+\Omega\left(y \frac{\partial L}{\partial z}\right)+\frac{\partial L}{\partial x}=0,
\end{aligned}
$$




$$
\begin{gathered}
\text { dif2. }\left[X \frac{\partial}{\partial x}+Y \frac{\partial}{\partial y}+Z \frac{\partial}{\partial z}\right]\left(\frac{\partial L}{\partial y}\right)+\frac{\partial L}{\partial y}=0, \\
\Omega\left(\frac{\partial L}{\partial y}\right)+\frac{\partial L}{\partial y}=0 .
\end{gathered}
$$

If we take the curve $\alpha$, for all equations, as an integral curve of $\Omega$ such that it is $\Omega(\alpha)=\dot{\alpha}=\frac{\partial}{\partial t}(\alpha)$

$$
\begin{aligned}
& \text { dif1. } \frac{\partial}{\partial t}\left(\frac{\partial L}{\partial x}\right)+\frac{\partial}{\partial t}\left(y \frac{\partial L}{\partial z}\right)+\frac{\partial L}{\partial x}=0 \\
& \operatorname{dif} 2 . \frac{\partial}{\partial t}\left(\frac{\partial L}{\partial y}\right)+\frac{\partial L}{\partial y}=0
\end{aligned}
$$

such that the differential equations (25) are named Euler-Lagrange equations on 3-dimensional real number space such that this is shown in the form of $(T M, g, \varphi)$. Additionally, therefore the triple $\left(T M, \Phi_{L}, \Omega\right)$ is called a Euler-Lagrangian mechanical system on $(T M, g, \varphi)$.

\section{Equations Solving with Computer and Graph of System}

The location of each object in space is represented by three dimensions in physical space. These three dimensions can be labelled by a combination of three chosen from the terms time, length, width, height, depth, mass, density, and breadth. (25) are partial differential equations and there are 4 independent variables on contact manifolds. Using Maple program, the solution of the equation system (25) is as follows:

$$
\begin{aligned}
L(x, y, z, t)= & F_{1}(t)+\exp (-t) * F_{2}(x)+F_{3}(z) \\
& +\exp (t) * F_{4}(y,(x * y-2 * z) / y) .
\end{aligned}
$$


Example 2. We draw a graph based on specific selected function for system (25)

$$
L(x, y, z, t)=t+\exp (-t) * x+z+\exp (t)
$$

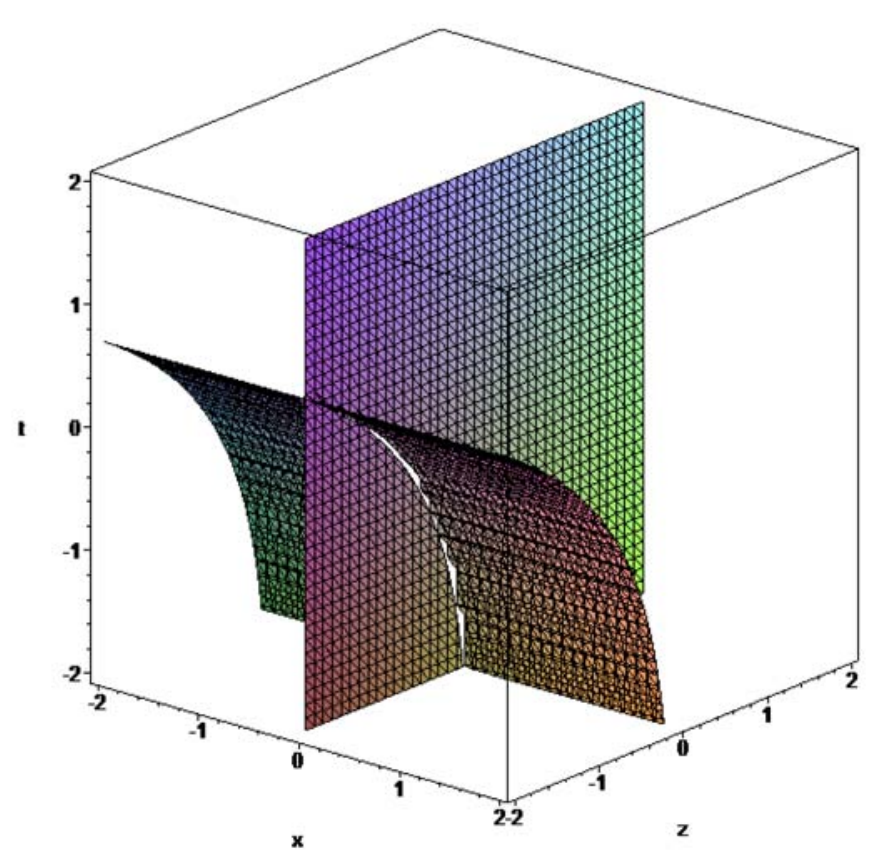

Figure 1.

\section{Conclusion}

It is well known that paracontact geometry is in many ways an odddimensional counterpart of symplectic geometry such that it belongs to the even-dimensional world. Both paracontact and symplectic geometry are motivated by the mathematical formalism of classical, analytical, and dynamical mechanics. Additionally, one can consider either the evendimensional phase space of a mechanical system or the odd-dimensional extended phase space that includes the time variable. Also, classical field theory utilizes traditionally the language of Euler-Lagrangian dynamics. This theory was extended to time-dependent classical mechanics. A 
Euler-Lagrange space has been certified as an excellent model for some important problems in relativity, gauge theory and electromagnetism. Euler-Lagrangian gives a model for both the gravitational and electromagnetic fields in a very natural blending of the geometrical structures of the space with the characteristic properties of these physical fields. Euler-Lagrangian dynamics is used as a model for field theory, quantum physics, optimal control, biology, and fluid dynamics [29-31].

Actually, our universe is three-dimensional such that Einstein added time as the fourth dimension. By this study, the above mentioned forms, we were transferred 3-dimensional real number space on an almost paracontact manifold for the mechanical system. The obtained timedependent equations system (25) on 3-dimensional real number space are very important to explain the rotational spatial mechanical-physical problems. In addition, in the equations closed solutions (26) were found using symbolic computation program and special election of the solution was drawn (Figure 1).

\section{Acknowledgement}

This work was supported by the agency BAP of Pamukkale University, project number: 2326. In addition, it orally was presented at the congress "3rd International Eurasian Conference on Mathematical Sciences and Applications, 25 th to 28 ${ }^{\text {th }}$ August 2014, Vienna, Austria”.

\section{References}

[1] M. M. Tripathi, E. Kilic, S. Y. Perktas and S. Keles, Indefinite almost paracontact metric manifolds, International Journal of Mathematics and Mathematical Sciences (2010), 1-19.

[2] S. Kr. Srivastava, D. Narain and K. Srivastava, Properties of $\varepsilon-S$ paracontact manifold, VSRD-TNTJ 2(11) (2011), 559-569.

[3] M. Girtu, An almost 2-paracontact structure on the cotangent bundle of a Cartan space, Hacettepe Journal of Mathematics and Statistics 33 (2004), 15-22. 
[4] M. Ahmad and J.-B. Jun, Submanifolds of an almost $r$-paracontact Riemannian manifold endowed with a semi-symmetric non-metric connection, Journal of The Chungcheong Mathematical Society 22(4) (2009), 653-664.

[5] I. Kupeli Erken, Some classes of 3-dimensional normal almost paracontact metric manifolds, Honam Mathematical J. 37(4) (2015), 457-468.

[6] I. Kupeli Erken, On normal almost paracontact metric manifolds of dimension 3, 30(5) (2015), 777-788.

[7] M. B. Cappelletti, I. Kupeli Erken and C. Murathan, Nullity conditions in paracontact geometry, Differential Geom. Appl. 30 (2012), 665-693.

[8] J. Welyczko, Slant curves in 3-dimensional normal almost paracontact metric manifolds.

http://arxiv.org/abs/1212.5839v1, 2012.

[9] I. Kupeli Erken and C. Murathan, A complete study of three-dimensional paracontact $(\kappa, \mu, \nu)$-spaces.

arxiv.org/abs/1305.1511v3, 2013.

[10] M. Manev and M. Staikova, On almost paracontact Riemannian manifolds of type $(n, n)$, Journal of Geometry 72(1-2) (2001), 108-115.

[11] K. P. Pandey and B. Tiwari, On indefinite almost paracontact metric manifold, International Mathematical Forum 6(22) (2011), 1071-1078.

[12] S. S. Shukla and U. S. Verma, Paracomplex paracontact pseudo-Riemannian submersions, Hindawi Publishing Corporation Geometry 2014 (2014), 1-12.

[13] Y. Gunduzalp and B. Sahin, Paracontact semi-Riemannian submersions, Turkish Journal of Mathematics 37 (2013), 114-128.

[14] A. Bucki, Product submanifold almost $r$-paracontact Riemannian manifold of $p$-Sasakian type, Soochov Journal of Mathematics 24(4) (1998), 255-259.

[15] B. E. Acet, E. Kilic and S. Y. Perktas, Some curvature conditions on a para-Sasakian manifold with canonical paracontact connection, International Journal of Mathematics and Mathematical Sciences 2012 (2012), 1-24.

[16] M. Ahmad, A. Haseeb, J.-B. Jun and S. Rahman, On almost r-paracontact Riemannian manifold with a certain connection, Commun. Korean Math. Soc. 25(2) (2010), 235-243.

[17] G. Nakova and S. Zamkovoy, Almost paracontact manifold.

arXiv:0806.3859v2, 2009.

[18] Z. Kasap and M. Tekkoyun, Mechanical systems on almost para/pseudo-KählerWeyl manifolds, IJGMMP 10(5) (2013), 1-8.

[19] Z. Kasap, Weyl-mechanical systems on tangent manifolds of constant W-sectional curvature, IJGMMP 10(10) (2013), 1-13. 
[20] M. Tekkoyun, On para-Euler-Lagrange and para-Hamiltonian equations, Phys. Lett. A 340 (2005), 7-11.

[21] S. Zamkovoy, Canonical connections on paracontact manifolds, Annals of Global Analysis and Geometry 36(1) (2009), 37-60.

[22] Y. Watanabe, Almost Hermitian and Kähler structures on product manifolds, Proceedings of the Thirteenth International Workshop on Diff.-Geom. 13 (2009), $1-16$.

[23] A. Newlander and L. Nirenberg, Complex analytic coordinates in almost complex manifolds, Ann. of Math. 65 (1957), 391-404.

[24] S. M. Salamon, Hermitian geometry.

http://calvino.polito.it/ salamon/G/bfs.pdf

[25] M. Falcitelli and A. Farinola, Almost-Hermitian geometry, Differential Geometry and its Applications 4 (1994), 259-282.

[26] D. McDu and D. Salamon, J-Holomorphic Curves and Quantum Cohomology, 1995.

[27] J. Klein, Escapes Variationnels et Mécanique, Ann. Inst. Fourier, Grenoble 12 (1962).

[28] A. Ibord, The geometry of dynamics, Extracta Mathematicable 11(1) (1996), 80-105.

[29] M. De Leon and P. R. Rodrigues, Methods of Differential Geometry in Analytical Mechanics, North-Hol. Math. St., 152, Elsevier Sc. Pub. Com. Inc., Amsterdam, 1989.

[30] R. Miron, D. Hrimiuc, H. Shimada and S. V. Sabau, The Geometry of Hamilton and Lagrange Spaces, Kluwer Academic Publishers, 2002.

[31] H. Weyl, Space-Time-Matter, Dover Publ. 1922, Translated from the 4th German Edition by H. Brose, 1952. 